\title{
GLOBAL AND APPROXIMATE GLOBAL OPTIMALITY OF MYOPIC ECONOMIC DECISIONS*
}

\author{
Leigh TESFATSION \\ University of Southern California, Los Angeles, CA 90007, USA
}

Received July 1979, final version received December 1979

\begin{abstract}
A general discrete-time stochastic control model is developed which encompasses many wellknown economic models. In the context of the general model, sufficient conditions are derived for the equivalence and approximate equivalence of myopic (sequential single-period) and global (simultaneous multi-period) expected return maximization. A bound provided for the global return loss resulting from myopic optimization is shown to vary directly with the degree of uncertainty and inversely with the degree of positive correlation in period-by-period returns. Characteristics of intermediate-period return functions which partially order them in terms of final-period expected return performance are clarified. Results are illustrated by portfolio and macro policy model examples.
\end{abstract}

\section{Introduction}

In theoretical models of dynamic resource allocation problems, the criterion function of the economic planner is commonly specified in the form of a return function integrated over the entire duration of the resource allocation process. Nevertheless, it has long been noted [Leontief (1958), Koopmans (1967), Day (1969), Simon (1971)] that in actual problem contexts the time horizon over which plans are formulated must generally be short in relation to the history of the process as a whole. Information regarding future technology, preferences, and general economic environment may be incomplete. Alternatively, the required calculations may be analytically intractable.

The question then arises whether the sequential selection of short-term limited foresight controls ever results in returns which are approximately optimal in some long-term sense. Day and Fan (1976), Kos (1971), and Keeler (1974) investigate the relation between short-term and long-term

\footnotetext{
*This material is based upon work supported by the National Science Foundation under Grant No. ENG77-28432. An earlier version of this paper was presented at the EconomicsMRG Research Seminar, USC, November 27, 1978, and at the Workshop in Mathematical Economic Theory and Econometrics, UCLA, November 30, 1978. The author is grateful to R.H. Day, Y. Haitovsky, and two anonymous referees for helpful comments.
} 
optimization for several deterministic growth models with consumption and terminal stock objectives, and Landau (1976) carries out a similar investigation for a deterministic pricing model with total profit objective. Mossin (1968) and Hakansson (1971) characterize the constant risk aversion utility functions for which short-term and long-term optimization coincide in the context of a portfolio model with terminal wealth objective. Finally, Aoki (1976), Bar-Shalom and Tse (1976), Chow (1975), and Norman (1976) propose several global return approximation schemes for dynamic macro policy models.

The present paper investigates the optimality of sequential single-period expected return maximization for a general class of stochastic discrete-time resource allocation models with multi-period expected return objectives. This class of models includes the standard dynamic macro policy model used by Aoki (1976) et al., as well as a variety of microeconomic models from production, consumption, and investment theory, e.g., the portfolio model used by Mossin (1968) and a version of the pricing model used by Landau (1976).

The basic idea of the paper is as follows. For some of the models under consideration, e.g., production and investment, period-by-period returns tend to be positively correlated. For others, e.g., consumption or consumptioninvestment, period-by-period returns tend either to be negatively correlated or to exhibit no consistent pattern. Intuitively, myopic (sequential singleperiod) expected return maximization should result in better global (multiperiod) expected return performance for the first type of model than for the second; and, for the first type of model, the resulting global expected return performance should be adversely affected by increased uncertainty and favorably affected by increased positive correlation in period-by-period returns.

An affirmative answer for these suppositions is provided below. Briefly, the basic resource allocation model is developed in section 2 . In section 3 it is shown that myopic expected return maximization results in optimal global expected return if period-by-period returns exhibit positive linear correlation. Linearity can be omitted if certain additional conditions are met. For the important special case in which all probability distributions are degenerate, linearity can be omitted without additional conditions. These results provide a systematic explanation for a variety of economic models in which myopic optimization yields globally optimal return, e.g., the constant risk aversion portfolio model of Mossin (1968) and a dynamic linear-quadratic policy model of Aoki (1967).

In section 4, after suitable distance functions are introduced for measuring uncertainty and positive correlation in returns, the global expected return loss associated with myopic expected return maximization is shown to be bounded above by terms which vary directly with the degree of uncertainty 
and inversely with the degree of positive correlation in period-by-period returns. Also, characteristics of intermediate-period return functions which partially order them in terms of final-period expected return performance are clarified.

Macro policy model and portfolio model examples of analytic findings are presented in section 5, and concluding comments are given in section 6 . Proofs of theorems are outlined in an appendix.

\section{The basic model}

Consider a finite-horizon economic system described by equations of the form

$$
\begin{aligned}
& x_{0}=\bar{x} \quad \text { (initial conditions), } \\
& x_{n+1} \doteq f_{n}\left(\omega_{n}, v_{n}, x_{n}\right), \quad 0 \leqq n \leqq N,
\end{aligned}
$$

where, for each $n \in\{0, \ldots, N\}$, the $n$th period system state $x_{n}$ is an element of an open set $X \subset R^{q}$, the $n$th period control $v_{n}$ is constrained to lie in an admissible control set $V\left(n, x_{n}\right) \subset V$ for some open set $V \subset R^{r}$, the $n$th period random disturbance $\omega_{n}$ is an element of a set $\Omega \subset R^{s}$, and $f_{n}: \Omega \times V \times X \rightarrow X$ is a continuous ${ }^{1}$ state function. Letting $\mathscr{F}$ denote the $\sigma$-algebra generated by the open sets of $\Omega$, it will be assumed that $\omega_{n}$ is governed by a transition probability $^{2} p_{n}\left(\cdot \mid v_{n}, x_{n}\right): \mathscr{F} \rightarrow R$ conditioned on the current time $n$, the current control selection $v_{n}$, and the current state $x_{n}$. In addition, it will be assumed that the value associated with each possible disturbance, control, and state configuration $\left(\omega_{n}, v_{n}, x_{n}\right)$ for period $n$ is measured by a continuous return function $W_{n}: \Omega \times V \times X \rightarrow R$.

An admissible feedback control law for the problem at hand is any vector $v$ $=\left(v_{0}(\cdot), \ldots, v_{N}(\cdot)\right)$ of measurable functions $v_{n}: X \rightarrow V$ satisfying $v_{n}(x) \in V(n, x)$ for each $x \in X$. The symbol $\mathscr{L}$ will be used to denote the set of all admissible feedback control laws $v$. The objective assumed for the economic planner will be either the maximization of final-period expected return

$$
E\left[W_{N}\left(\omega_{N}, v_{N}\left(x_{N}\right), x_{N}\right) \mid v, \bar{x}\right],
$$

\footnotetext{
'It is assumed throughout the paper that $X, V$, and $\Omega$ have the relative topology with respect to Euclidean $q$-space, $r$-space, and $s$-space, respectively, and that products of $X, V$, and $\Omega$ have the corresponding product topology. Each of the spaces $X, V$, and $\Omega$ will also be regarded as a measurable space, with $\sigma$-algebra generated by its open sets.

${ }^{2}$ More precisely, it is assumed that $p_{n}(\cdot \mid v, x): \mathscr{F} \rightarrow R$ is a probability measure for each $(v, x) \in V$ $\times X$, and $p_{n}(A \mid \cdot): V \times X \rightarrow R$ is a measurable function for each $A \in \mathscr{F}$.
} 
or the maximization of total expected return

$$
E\left[\sum_{n=0}^{N} W_{n}\left(\omega_{n}, v_{n}\left(x_{n}\right), x_{n}\right) \mid \boldsymbol{v}, \bar{x}\right]
$$

via selection of a feedback control law $v \in \mathscr{L}^{3}$

For brevity, any optimization problem meeting the above specifications will be referred to as a basic model. ${ }^{4}$ As will be illustrated in section 5 , the basic model encompasses a wide variety of interesting economic optimization problems.

In section 3 it will at times also prove useful to consider a special basic model for which the state space $X$ is an open subset of $R$, and the decision maker has a final-period expected return objective function (2) with finalperiod return function $W_{N}: \Omega \times V \times X \rightarrow R$ given by $W_{N}(\omega, v, x) \equiv \varphi \circ f_{N}(\omega, v, x)$ $=\varphi\left(x_{N+1}\right)$ for some continuous strictly increasing function $\varphi: X \rightarrow R$. Moreover, for the special basic model the state functions $f_{n}(\omega, v, x)$ will be assumed to be strictly increasing in $x$, and the transition probabilities $p_{n}(\cdot \mid v, x)$ will be assumed to be non-degenerate measures independent of $v$ and $x$. The following additional restrictions will be imposed as needed:

(A.1) $\varphi(x)$ is twice continuously differentiable, and $f_{n}(\omega, v, x)$ is twice continuously differentiable in $v$ and $x$ for each $\omega \in \Omega$ and $n \in\{0, \ldots, N\}$.

(A.2) $\Omega$ is compact. ${ }^{5}$

${ }^{3}$ The expectation operator $E[\cdot \mid v, x]$ is more precisely defined as follows. Let $\Omega^{N}$ denote the set of all disturbance sequences $\omega^{N}=\left(\omega_{0}, \ldots, \omega_{N}\right)$ satisfying $\omega_{n} \in \Omega, 0 \leqq n \leqq N$, and let $\mathscr{F}^{N}$ denote the product $\sigma$-algebra generated hy all cylinder sets of the form

$$
\prod_{n-0}^{N} A_{n}=\left\{\omega^{N} \in \Omega^{N} \mid \omega_{0} \in A_{0}, \ldots, \omega_{N} \in A_{N}\right\}
$$

where $A_{n} \in \mathscr{F}, 0 \leqq n \leqq N$. Finally, for each $v \in \mathscr{L}$ and $x \in X$, let $p^{N}(\cdot \mid v, x)$ denote the unique probability measure on $\left(\Omega^{N}, \mathscr{F}^{N}\right)$ satisfying

$$
p^{N}\left(\prod_{n=0}^{N} A_{n} \mid v, x\right)=\iint_{\lambda_{0} l_{1} \ldots} \ldots \int_{\lambda_{N}} p_{n}\left(\mathrm{~d} \omega_{N} \mid v_{n}\left(x_{n}\right), x_{n}\right) \ldots p_{1}\left(\mathrm{~d} \omega_{1} \mid v_{1}\left(x_{1}\right), x_{1}\right) p_{0}\left(\mathrm{~d} \omega_{0} \mid v_{0}(x), x\right),
$$

for each cylinder set $\Pi_{n=0}^{N} A_{n} \in \bar{F}^{N}$ [see Hinderer (1971, thm. A.5, p. 148)]. Expectation with respect to $\left\langle\Omega^{N}, \mathscr{F}^{N}, p^{N}(\cdot \mid v, x)\right\rangle$ is then denoted by $E[\cdot \mid v, x]$.

${ }^{4} \mathrm{An}$ axiomatization for a one-period version of the basic model with discrete probability distributions is provided in Tesfatsion (to appear), where it is shown that the basic model generalizes the Savage expected utility model, the Marschak-Radner team model, and the standard Bayesian statistical decision model. The symmetrical treatment of return and probability in the basic model has proved to be useful in the development of a new approach to adaptive control, direct criterion function updating. See Tesfatsion $(1978,1979)$.

${ }^{5}$ The sole purpose of the compact $\Omega$ restriction is to allow the interchange of expectation and differentiation operations. Various alternative restrictions would serve equally well for this purpose. 
(A.3) A unique regular interior ${ }^{6}$ optimal feedback control law $v^{0}$ $=\left(v_{0}^{\circ}(\cdot), \ldots, v_{N}^{o}(\cdot)\right)$ exists.

(A.4) $\varphi(x)$ is thrice continuously differentiable.

(A.5) The state functions $f_{n}: \Omega \times V \times X \rightarrow X$ are given by $f_{n}(\omega, v, x) \equiv b_{n} x$ $+Q_{n}(\omega, v)$ for some constants $b_{n} \in R_{++}$and continuous functions $Q_{n}$ : $Q \times V \rightarrow R$ thrice continuously differentiable in $v$.

\section{Sufficient conditions for myopic and global expected return equivalence}

Let $E_{n}[\cdot \mid v, x]$ denote expectation with respect to the $n$th period transition probability $\left\langle\Omega, \mathscr{F}, p_{n}(\cdot \mid v, x)\right\rangle$, and consider the three alternative expected return objectives

$$
\begin{aligned}
& \text { (Myopic) } \max _{v_{n} \in V\left(n, x_{n}\right)} E_{n}\left[W_{n}\left(\omega_{n}, v_{n}, x_{n}\right) \mid v_{n}, x_{n}\right], \quad 0 \leqq n \leqq N, \\
& \text { (Final-Period) } \max _{v \in \mathscr{P}} E\left[W_{N}\left(\omega_{N}, v_{N}\left(x_{N}\right), x_{N}\right) \mid v, \bar{x}\right], \\
& \text { (Total) } \max _{\boldsymbol{v} \in \mathscr{\Psi}} E\left[\sum_{n=0}^{N} W_{n}\left(\omega_{n}, v_{n}\left(x_{n}\right), x_{n}\right) \mid v, \bar{x}\right] .
\end{aligned}
$$

For each $n \in\{0, \ldots, N\}$ and $x \in X$, let $F_{n}(x)$ denote the maximum attainable final-period expected return beginning in period $n$ with initial state $x$, and using feedback control. Then [Hinderer $(1971$, thm. 14.4, p. 101; pp. 104105; thm. 17.6, p. 111)].

$$
\begin{aligned}
& F_{N}(x)=\sup _{v \in V(N, x)} E_{N}\left[W_{N}(\omega, v, x) \mid v, x\right], \\
& F_{n}(x)=\sup _{v \in V(n, x)} E_{n}\left[F_{n+1} \circ f_{n}(\omega, v, x) \mid v, x\right], \quad 0 \leqq n \leqq N-1,
\end{aligned}
$$

and a feedback control law $v^{\circ} \in \mathscr{L}$ satisfies the final-period expected return objective (5) if and only if it satisfies the dynamic programming optimality equations

$$
\begin{aligned}
& F_{N}\left(x_{N}\right)=E_{N}\left[W_{N}\left(\omega_{N}, v_{N}^{o}\left(x_{N}\right), x_{N}\right) \mid v_{N}^{o}\left(x_{N}\right), x_{N}\right], \\
& F_{n}\left(x_{n}\right)=E_{n}\left[F_{n+1} \circ f_{n}\left(\omega_{n}, v_{n}^{o}\left(x_{n}\right), x_{n}\right) \mid v_{n}^{o}\left(x_{n}\right), x_{n}\right],
\end{aligned}
$$

$$
0 \leqq n \leqq N-1,
$$

${ }^{6}$ An optimal admissible feedback control law $v^{\circ}=\left(v_{0}^{0}(\cdot), \ldots, v_{N}^{0}(\cdot)\right)$ will be called regular interior if each component solution $v_{n}^{\circ}(x)$ to $\max E_{n}\left[F_{n+1} \circ f_{n} \mid v, x\right] \equiv Q(v, x)$ over $v \in V(n, x)$ satisfies $Q_{1}\left(v_{n}^{\circ}(x), x\right)=0$ and $Q_{r v}\left(v_{n}^{\circ}(x), x\right)$ negative definite, where $F_{n+1}$ is as defined in section 3 . Alternate sets of weak sufficient conditions guaranteeing the existence of an optimal admissible feedback control law for the basic model can be derived using the results of Hinderer (1971), Leland (1972), and Hildreth (1974). 
for almost every disturbance sequence $\left(\omega_{0}, \ldots, \omega_{N}\right){ }^{7}$ Thus a feedback control law $v^{*} \in \mathscr{L}$ which satisfies the myopic expected return objective (4) provides an optimal feedback control law for the final-period expected return objective (5) if and only if

$$
F_{n}\left(x_{n}\right)=E_{n}\left[F_{n+1} \circ f_{n}\left(\omega_{n}, v_{n}^{*}\left(x_{n}\right), x_{n}\right) \mid v_{n}^{*}\left(x_{n}\right), x_{n}\right], \quad 0 \leqq n \leqq N-1,
$$

for almost every sequence $\left(\omega_{0}, \ldots, \omega_{N}\right)$.

Similarly, letting $T_{n}(x)$ denote the maximum attainable total expected return beginning in period $n$ with initial state $x$ and using feedback control, it follows [Hinderer (1971, op. cit.)] that

$$
\begin{aligned}
& T_{N}(x)=\sup _{v \in V(N, x)} E_{N}\left[W_{N}(\omega, v, x) \mid v, x\right], \\
& T_{n}(x)=\sup _{v \in V(n, x)} E_{n}\left[W_{n}(\omega, v, x)+T_{n+1} \circ f_{n}(\omega, v, x) \mid v, x\right], \quad 0 \leqq n \leqq N-1,
\end{aligned}
$$

and a feedback control law $\boldsymbol{v}^{0} \in \mathscr{L}$ satisfies the total expected return objective (6) if and only if it satisfies the corresponding optimality equations

$$
\begin{aligned}
& T_{N}\left(x_{N}\right)=E_{N}\left[W_{N}\left(\omega_{N}, v_{N}^{\circ}\left(x_{N}\right), x_{N}\right) \mid v_{N}^{\circ}\left(x_{N}\right), x_{N}\right], \\
& T_{n}\left(x_{n}\right)=E_{n}\left[W_{n}\left(\omega_{n}, v_{n}^{\circ}\left(x_{n}\right), x_{n}\right)+T_{n+1} \circ f_{n}\left(\omega_{n}, v_{n}^{\circ}\left(x_{n}\right), x_{n}\right) \mid v_{n}^{\circ}\left(x_{n}\right), x_{n}\right], \\
& 0 \leqq n \leqq N-1,
\end{aligned}
$$

for almost every disturbance sequence $\left(\omega_{0}, \ldots, \omega_{N}\right)$. Thus a feedback control law $v^{*} \in \mathscr{L}$ which satisfies the myopic expected return objective (4) provides an optimal feedback control law for the total expected return objective (6) if and only if

$$
\begin{gathered}
T_{n}\left(x_{n}\right)=E_{n}\left[W_{n}\left(\omega_{n}, v_{n}^{*}\left(x_{n}\right), x_{n}\right)+T_{n+1} \circ f_{n}\left(\omega_{n}, v_{n}^{*}\left(x_{n}\right), x_{n}\right) \mid v_{n}^{*}\left(x_{n}\right), x_{n}\right], \\
0 \leqq n \leqq N-1 .
\end{gathered}
$$

Given degenerate probability distributions, equality (9) holds for $v^{*} \in \mathscr{L}$ satisfying (4) if and only if maximum future expected return $F_{n+1} \circ f_{n}\left(\omega_{n}, \cdot, x_{n}\right)$ and currently realized return $W_{n}\left(\omega_{n}, \cdot, x_{n}\right)$ attain a maximum at the same

\footnotetext{
${ }^{7}$ More precisely, using the definitions presented in footnote 3, the optimality equations must hold for $p^{N}$-almost every disturbance sequence $\omega^{N}-\Omega^{N}$. The symbol o denotes function composition, e.g., $h \circ S(x) \equiv h(S(x))$.
} 
control selection $v \in V\left(n, x_{n}\right)$, regardless of how dissimilar $F_{n+1} \circ f_{n}$ and $W_{n}$ may be in other respects. A sufficient condition for $F_{n+1} \circ f_{n}$ and $W_{n}$ to have a common maximizing point is that $F_{n+1} \circ f_{n}$ be an increasing function of $W_{n}$ in the sense that

$$
F_{n+1} \circ f_{n}=H_{n+1} \circ W_{n}
$$

for some function $H_{n+1}: R \rightarrow R$ with $H_{n+1}^{\prime}>0$. If for each $v \in V\left(n, x_{n}\right)$ the correlation coefficient

$$
\rho^{v}\left(W_{n}, F_{n+1} \circ f_{n}\right) \equiv \frac{\operatorname{cov}\left(W_{n}\left(\omega, v, x_{n}\right), F_{n+1} \circ f_{n}\left(\omega, v, x_{n}\right)\right)}{\left\{\operatorname{var}\left(W_{n}\left(\omega, v, x_{n}\right)\right) \operatorname{var}\left(F_{n+1} \circ f_{n}\left(\omega, v, x_{n}\right)\right)\right\}^{\frac{1}{2}}},
$$

for the two random variables $W_{n}\left(\cdot, v, x_{n}\right)$ and $F_{n+1} \circ f_{n}\left(\cdot, v, x_{n}\right)$ is well-defined, then condition (13) guarantees [Hildreth-Tesfatsion (1977, thm. 1)] that $\rho^{v}\left(W_{n}, F_{n+1} \circ f_{n}\right) \in(0,1]$, i.e., that current and future returns are positively correlated for each admissible control selection $v$. Although the presence of non-degenerate expectation operators in (9) and (12) complicates matters, a generalized version of condition (13) and an analogous condition for $T_{n+1} \circ f_{n}$ will play fundamental roles throughout the remaining sections of the paper.

The first two theorems, below, provide sufficient conditions for myopic objective (4) solutions to yield optimal feedback control laws for the global objectives (5) and (6), and conversely. As a direct corollary, they also provide sufficient conditions for the return-to-go-functions $F_{n+1} \circ f_{n}$ and $T_{n+1} \circ f_{n}$ in the optimality equations (8) and (11) to be approximated by simpler expressions (e.g., truncated Taylor's expansions) without loss of control performance. $^{8}$ Examples of economic models satisfying the hypotheses of these theorems are presented in section 5 .

\footnotetext{
${ }^{8}$ For example, suppose the problem is to determine whether the return-to-go terms $T_{n+1} \circ f_{n}$ in the optimality equations (11) can be approximated by certain simpler expressions $S_{n+1} \circ f_{n}$ without loss of return performance, the control objective being to maximize total expected return. Replacing each single-period return function $W_{n}$ by $W_{n}^{*}(\omega, v, x) \equiv W_{n}(\omega, v, x)$ $+\left[S_{n+1} \circ f_{n}\right](\omega, v, x)-S_{n}(x)$, with $S_{N+1}(\cdot) \equiv S_{0}(\cdot) \equiv 0$, this problem is equivalent to determining whether the myopic sequential maximization of 'current' expected return $E_{n}\left[W_{n}^{\prime}\left(\omega, v, x_{n}\right) \mid v, x_{n}\right]$ results in a control law $v^{*}$ which satisfies the optimality equations $(11)$ with $W_{0}, \ldots, W_{N}$ in place of $W_{0}, \ldots, W_{N}$. Any such control law would also be optimal for the original problem, since

$$
E\left[\sum_{n=0}^{N} W_{n}^{\cdot}\left(w_{n}, v_{n}\left(x_{n}\right), x_{n}\right) \mid v, \bar{x}\right]=E\left[\sum_{n=0}^{N} W_{n}\left(w_{n}, v_{n}\left(x_{n}\right), x_{n}\right) \mid v, \bar{x}\right],
$$

for each $v \in \mathscr{L}$. Similarly, in considering approximations $S_{n+1} \circ f_{n}$ for the return-to-go terms $F_{n+1} \circ f_{n}$ in the optimality equations (8), one can apply the sufficiency conditions for the equivalence of objectives (4) and (5) with each original return function $W_{n}$ replaced by $W_{n}^{\prime}$ $\equiv S_{n+1} \circ f_{n}$.

Various return-to-go approximations have been suggested. See Aoki (1976, ch. VII), Aoki (1967, ch. 10), Bar-Shalom and Tse (1976), Chow (1975, ch. 10-12), and Norman (1976).
} 
Theorem 3.1. Suppose for each $n \in\{0, \ldots, N-1\}$ the return-to-go function $F_{n+1} \circ f_{n}$ in the optimality equation (8b) has the form

$$
\left[F_{n+1} \circ f_{n}^{\prime}\right](\omega, v, x)=a_{n}(x)\left[H_{n+1} \circ W_{n}\right](\omega, v, x)+b_{n}(x),
$$

for some functions $a_{n}: X \rightarrow R_{++}, b_{n}: X \rightarrow R$, and $H_{n+1}: R \rightarrow R$, where $H_{n+1}$ is strictly increasing. ${ }^{9}$ Then any one of the following five restriction sets guarantees that a feedback control law $v^{*} \in \mathscr{L}$ satisfies the myopic expected return objective (4) if' and only if it is also an optimal jeedback control law for the final-period expected return objective (5).

(1) For each $n \in\{0, \ldots, N-1\}$, the function $H_{n+1}(\cdot)$ is linear.

(2) (Deterministic control). For each $n \in\{0, \ldots, N-1\}$, the probability distributions $\left\langle\Omega, \mathscr{F}, p_{n}\left(\cdot \mid v, x_{n}\right)\right\rangle, v \in V\left(n, x_{n}\right)$, are degenerate.

(3) For each $n \in\{0, \ldots, N-1\}$, the return function $W_{n}(\cdot)$ has the form $W_{n}(\omega, v, x) \equiv S_{n}(v, x)$.

(4) For each $n \in\{0, \ldots, N-1\}$, the probability measure $p_{n}\left(\cdot \mid v, x_{n}\right)$ is independent of the control $v$; and the return function $W_{n}(\cdot)$ has the form $W_{n}(\omega, v, x) \equiv Q_{n}(\omega, x)+Z_{n}(v, x)$.

(5) For each $n \in\{0, \ldots, N-1\}$, the nth period disturbance $\omega_{n}$ is a function of the nth period control $v_{n}$ and nth period state $x_{n}$ of the form $\omega_{n}=g_{n}\left(v_{n}, x_{n}\right)$ $+\delta_{n}$, where $\left(\delta_{n}\right)$ is a serially uncorrelated process; and the return function $W_{n}(\cdot)$ has the form $W_{n}(\omega, v, x) \equiv Q_{n}(x) \omega+Z_{n}(v, x)$.

If for each $n \in\{0, \ldots, N-1\}$ the probability distribution $p_{n}\left(\cdot \mid v, x_{n}\right)$ is independent of the selected control $v$, then the functions $b_{n}(\cdot)$ in condition (15) may depend on $\omega$ without affecting the above conclusions.

The next theorem follows from Theorem 3.1 by straightforward modifications.

Theorem 3.2. Suppose for each $n \in\{0, \ldots, N-1\}$ the return-to-go function $T_{n+1} \circ f_{n}$ in the optimality equation (11b) has the form

$$
\left[T_{n+1} \circ f_{n}\right](\omega, v, x)=a_{n}(x)\left[H_{n+1} \circ W_{n}\right](\omega, v, x)+b_{n}(x),
$$

for some functions $a_{n}: X \rightarrow R_{+}, b_{n}: X \rightarrow R$, and $H_{n+1}: R \rightarrow R$, where $H_{n+1}$ is nondecreasing. Then any one of the restriction sets $(1)-(5)$ listed in Theorem 3.1 guarantees that a feedback control law $v^{*} \in \mathscr{L}$ satisfies the myopic expected return obiective (4) if and only if it is also an optimal feedback control law for the totan expected return objective (6). If for each $n \in\{0, \ldots, N-1\}$ the

\footnotetext{
${ }^{9}$ As usual, we define $R_{+} \equiv\{z \in R \mid z \geqq 0\}$ and $R_{+_{+}} \equiv\{z \in R \mid z>0\}$.
} 
probability distribution $p_{n}\left(\cdot \mid v, x_{n}\right)$ is independent of the selected control $v$, then the functions $b_{n}(\cdot)$ in (16) may depend on $\omega$ without affecting the above conclusions.

As the following Corollary 3.3 demonstrates, the three return objectives (4), (5), and (6) yield identical optimal control laws if maximum expected return for each period $n+1$ is a positive linear affine function of the return realized in the previous period $n$. All previously noted equivalences of myopic and global optimization known to this author are special cases of Corollary 3.3. (See section 5.)

Corollary 3.3. Suppose for each $n \in\{0, \ldots, N-1\}$ the maximum expected return

$$
M_{n+1}(x) \equiv \max _{v \in \mathcal{V}(n+1, x)} E_{n+1}\left[W_{n+1}(\omega, v, x) \mid v, x\right]
$$

for period $n+1$ beginning in state $x$ is a positive linear affine function of the return realized in the previous period $n$ in the sense that

$$
\left[M_{n+1} \circ f_{n}\right](\omega, v, x)=c_{n}(x) W_{n}(\omega, v, x)+d_{n}(x),
$$

for some functions $c_{n}: X \rightarrow R_{+}$and $d_{n}: X \rightarrow R$. Then an optimal jeedback control law for any: one of the three expected return objectives (4), (5), or (6) yields an optimal feedback control law for the remaining two objectives. The equivalence still holds for objectives (4) and (6) if the functions $c_{n}(x)$ take on zero values. If for each $n \in\{0, \ldots, N-1\}$ the distribution $p_{n}\left(\cdot \mid v, x_{n}\right)$ is independent of the selected control $v$, then the functions $d_{n}(\cdot)$ in (18) may depend on $\omega$ without affecting the above conclusions.

Consider the special basic model with additional restrictions (A.1)-(A.5), outlined at the end of section 2 . Let

$$
G(v) \equiv E_{n}\left[F_{n+1} \circ f_{n}(\omega, v, x) \mid v, x\right]
$$

denote the maximum attainable final-period expected return starting in period $n$ with initial state $x$ and arbitrary control selection $v \in V(n, x)$. By assumption (A.3), the optimal control selection $v_{n}^{\circ}(x)$ for period $n$ in state $x$, the maximizer of $G(v)$ over $V(n, x)$, is assumed to exist. The next theorem demonstrates that a certain myopically selected control $v_{n}^{*}(x)$ yields the globally optimal return $G\left(v_{n}^{0}(x)\right)$ if the final-period utility function $\varphi(\cdot)$ exhibits constant absolute risk aversion. We thus obtain a generalization of a portfolio model result of Mossin (1968). 
Lemma 3.4. For any special basic model satisfying assumptions (A.1)-(A.3), the return-to-go functions $F_{n}: X \rightarrow R$ are well-defined strictly increasing twice differentiable functions satisfying the following relationships for each $n \in\{0, \ldots, N\}:$ Letting $F_{N+1}(\cdot) \equiv \varphi(\cdot), T$ denote transpose, and d denote the point $\left(\omega, v_{n}^{\circ}(x), x\right)$,

$$
\begin{aligned}
& E_{n}\left[F_{n+1}^{\prime}\left(f_{n}(d)\right) \frac{\partial f_{n}}{\partial v}(d)\right]=\left(\begin{array}{c}
0 \\
\vdots \\
0
\end{array}\right), \\
& F_{n}^{\prime}(x)=E_{n}\left[F_{n+1}^{\prime}\left(f_{n}^{\prime}(d)\right) \frac{\partial f_{n}}{\partial x}(d)\right]>0, \\
& F_{n}^{\prime \prime}(x)=E_{n}\left[F_{n+1}^{\prime \prime}\left(f_{n}(d)\right)\left(\frac{\partial f_{n}}{\partial x}(d)\right)^{2}+F_{n+1}^{\prime}\left(f_{n}(d)\right) \frac{\partial^{2} f_{n}}{\partial x^{2}}(d)\right] \\
&+S_{n}(x)^{r \frac{r^{2} v_{n}^{o}(x)}{\partial x}},
\end{aligned}
$$

where the nth period optimal feedback control $v_{n}^{o}(x)$ is a continuously differentiable function of $x$ satisfying

$$
\left[\frac{\partial v_{n}^{\circ}}{\partial x}(x)\right]_{r \times 1}=-D_{n}(x)^{-1} S_{n}(x)
$$

with

$$
\begin{aligned}
{\left[S_{n}(x)\right]_{r \times 1} \equiv } & E_{n}\left[F_{n+1}^{\prime \prime}\left(f_{n}(d)\right) \frac{\partial f_{n}}{\partial x}(d) \frac{\partial f_{n}}{\partial v}(d)\right. \\
& \left.+F_{n+1}^{\prime}\left(f_{n}(d)\right) \frac{\partial^{2} f_{n}}{\partial x \partial v}(d)\right]
\end{aligned}
$$

and $D_{n}(x)$ a negative definite matrix given by

$$
\begin{aligned}
{\left[D_{n}(x)\right]_{r \times r} \equiv } & E_{n}\left[F_{n+1}^{\prime \prime}\left(f_{n}(d)\right) \frac{\partial f_{n}}{\partial v}(d) \frac{\partial f_{n}^{T}}{\partial v}(d)\right. \\
& \left.+F_{n+1}^{\prime}\left(f_{n}(d)\right) \frac{\partial^{2} f_{n}}{\partial v^{2}}(d)\right] .
\end{aligned}
$$

Theorem 3.5. Consider a special basic model satisfying assumptions (A.1)(A.5). Suppose $R_{\varphi}^{\prime}(x)=0$ for all $x \in X$, where $R_{\varphi}(x) \equiv-\varphi^{\prime \prime}(x) / \varphi^{\prime}(x)$ denotes the Pratt-Arrow measure of absolute risk aversion [Pratt (1964)]. If a control 
$v_{n}^{*}(x)$ is selected in period $n$ which solves

$$
\max _{v \in V(n, x)} E_{n}\left[U \circ f_{n}(\omega, v, x) \mid v, x\right],
$$

where $U: X \rightarrow R$ is defined by $U(x) \equiv \varphi\left(x \Pi_{j=1}^{N-n} b_{n+j}\right)$, then no global return loss results; i.e., $G\left(v_{n}^{*}(x)\right)=G\left(v_{n}^{0}(x)\right)$.

As is clear from the proofs of Corollary 3.3 and Theorem 3.5, the hypotheses of these propositions guarantee that conditions (15) and (16) in Theorems 3.1 and 3.2 are satisfied by positive linear affine functions $H_{n+1}$ : $R \rightarrow R$. Hence, current and future expected returns are perfectly positively correlated; for the correlation coefficient (14) takes on its maximum value 1.0 if and only if

$$
\left[F_{n+1} \circ f_{n}\right]\left(\cdot, v, x_{n}\right)=a_{n}\left(x_{n}\right) W_{n}\left(\cdot, v, x_{n}\right)+b_{n}\left(x_{n}\right) \quad p_{n}\left(\cdot \mid v, x_{n}\right)-\text { a.s., }
$$

for some constants $a_{n}\left(x_{n}\right)$ and $b_{n}\left(x_{n}\right)$, with $a_{n}\left(x_{n}\right)>0$ [Wilkes (1962, 3.4.3, p. 79)]. Similar observations hold for $W_{n}, T_{n+1} \circ f_{n}$, and $\rho^{v}\left(W_{n}, T_{n+1} \circ f_{n}\right)$ in the context of Theorem 3.2.

The final result of this section, Theorem 3.6, provides weaker hypotheses for the basic model which guarantee conditions (15) and (16) hold with possibly non-linear functions $H_{n+1}$. The remaining conditions for Theorems 3.1 and 3.2 are then easily checked by direct inspection. As will be indicated in section 5. the hypotheses of Theorem 3.6 are satisfied by sereral wellknown economic models.

Theorem 3.6. Suppose the following restrictions hold for the basic model for each $n \in\{0, \ldots, N\}$ :

(1) The state function $f_{n}: \Omega \times V \times X \rightarrow X$ satisfies $f_{n}(\omega, v, x)=\left[g_{n} \circ W_{n}\right](\omega, v, x)$ for some non-decreasing function $g_{n}: R \rightarrow X$.

(2) Either the transition probability $p_{n}(\cdot \mid v, x)$ is independent of the state $x$ for each $v \in V$; or the disturbance space $\Omega$ is a subset of $R$, and the return function $W_{n}(\omega, v, x)$ is non-decreasing in $\omega$ for each $(v, x) \in V \times X .^{10}$

(3) If $x^{\prime}>x^{\prime \prime}$ for some $x^{\prime}, x^{\prime \prime} \in X$, then $p_{n}\left(\cdot \mid v, x^{\prime \prime}\right)$ stochastically dominates $p_{n}\left(\cdot \mid v, x^{\prime}\right)$ for every $v \in V$; i.e.,

$$
\int_{\Omega\left(\omega^{*}\right)} p\left(\mathrm{~d} \omega \mid v, x^{\prime \prime}\right) \leqq \int_{\Omega(\omega *} p_{n}\left(\mathrm{~d} \omega \mid v, x^{\prime}\right)
$$

${ }^{10}$ The hypothesis $\Omega \subset R$ can be weakened to $\Omega \subset R^{s}, s \geqq 1$, if the stochastic dominance restriction (3) is strengthened to include additional restrictions on marginal distributions [Levy et al. (1974)]. 
for all $\omega^{*} \in \Omega$, where $\Omega\left(\omega^{*}\right) \equiv\left\{\omega \in \Omega \mid \omega \leqq \omega^{*}\right\}$.

(4) The return function $W_{n}(\omega, v, x)$ is non-decreasing in $x$ for each $(\omega, v) \in \Omega$ $\times V$.

(5) The admissible control set $V(n, x)$ is non-decreasing in $x$ in the sense that $V\left(n, x^{\prime \prime}\right) \supseteqq V\left(n, x^{\prime}\right)$ whenever $x^{\prime \prime} \geqq x^{\prime}$.

Then, for each $n \in\{0, \ldots, N-1\}$, the return-to-go functions $F_{n+1}: X \rightarrow R$ and $T_{n+1}: X \rightarrow R$ are non-decreasing, and similarly for the functions $H_{n+1}^{*}: R \rightarrow R$ and $H_{n+1}^{* *}: R \rightarrow R$ defined by $H_{n+1}^{*} \equiv\left[F_{n+1} \circ g_{n}\right]$ and $H_{n+1}^{* *} \equiv\left[T_{n+1} \circ g_{n}\right]$. If 'nondecreasing' is replaced by 'strictly increasing' in restriction (1) above, and the return function $W_{n}(\cdot)$ is assumed to be strictly increasing with respect to $x$ in (4), then the functions $F_{n+1}, T_{n+1}, H_{n+1}^{*}$, and $H_{n+1}^{* *}$ will be strictly increasing.

Remark. The positive correlation conditions (15) and (16) in Theorems 3.1 and 3.2 are satisfied under restriction (1) with $F_{n+1} \circ f_{n}=H_{n+1}^{*} \circ W_{n}$ and $T_{n+1} \circ f_{n}=H_{n+1}^{* *} \circ W_{n}$. The stochastic dominance restriction (3) implies that the $n$th period disturbance $\omega_{n}$ is positively correlated with the $n$th period initial state $x_{n}$.

\section{Approximate global optimality of myopic expected return maximization}

Consider an economic planner in period $n$ of a basic model resource allocation problem for which the current state is $x$. Suppose the planner wishes to maximize final-period expected return (2), and current return is positively correlated with final-period expected return in the sense that condition (15) holds. ${ }^{11}$ Then, for any admissible control selection $v \in V(n, x)$ for period $n$, the maximum attainable final-period expected return is

$$
\begin{aligned}
G(v) & \equiv E_{n}\left[F_{n+1} \circ f_{n}(\omega, v, x) \mid v, x\right] \\
& =a_{n}(x) E_{n}\left[H_{n+1} \circ W_{n}(\omega, v, x) \mid v, x\right]+b_{n}(x),
\end{aligned}
$$

where $a_{n}(x) \in R_{++}, b_{n}(x) \in R$, and $H_{n+1}: R \rightarrow R$ is strictly increasing.

The first problem considered in the present section is as follows: Suppose $v^{\circ} \equiv v_{n}^{\circ}(x)$ is a globally optimal admissible control selection for period $n$ in

\footnotetext{
"Unless otherwise stated, it is implicitly assumed throughout section 4 that an optimal admissible feedback control law $v^{\circ}$ exists for the basic model under discussion, hence the returnto-go functions $F_{n}$ are well-defined. In addition, it is generally assumed without comment that the functions $F_{n}$ have suitable differentiability properties, a well-established tradition in the economic control literature since general sufficient conditions guaranteeing differentiability of such functions have not yet been obtained [see Pitchford et al. (1977)]. Sufficient conditions guaranteeing differentiability are established in Lemma 3.4 for the special basic model.
} 
the sense that

$$
G\left(v^{\circ}\right)=\max _{v \in V(n, x)} G(v),
$$

and suppose $v^{*} \equiv v_{n}^{*}(x)$ is a myopically optimal admissible control selection for period $n$ in the sense that $v^{*}$ maximizes only current expected return, i.e.,

$$
E_{n}\left[W_{n}\left(\omega, v^{*}, x\right) \mid v^{*}, x\right]=\max _{v \in V(n, x)} E_{n}\left[W_{n}(\omega, v, x) \mid v, x\right]
$$

What is the loss in global expected return performance which results from using $v^{*}$ in place of $v^{\circ}$; i.e., what is the magnitude of the (non-negative) return loss

$$
G\left(v^{\circ}\right)-G\left(v^{*}\right)
$$

As will be shown in Theorem 4.2, below, the return loss (28) is bounded above by terms which vary directly with the degree of uncertainty and inversely with the degree of positive correlation in period-by-period returns. Using similar arguments, analogous results can easily be obtained for the return loss resulting from use of the myopic control $v^{*}$ in place of the control $v^{\circ}$ which is optimal for the total expected return objective (6).

A natural choice for measuring uncertainty in current ( $n$th period) return is maximum variance. Formally, letting

$$
\left.M(v) \equiv E_{n}\left[W_{n}(v), v, x\right) \mid v, x\right]
$$

denote the current expected return, and

$$
\sigma^{j}(v) \equiv E_{n}\left[\left(W_{n}(u, v, x)-M(v)\right)^{j} \mid v, x\right]
$$

denote the $j$ th central moment for current return associated with any control selection $v$, uncertainty in current return will be measured by

$$
\sigma^{2} \equiv \sup _{v \in V(n, x)} \sigma^{2}(v)
$$

As noted in section 3 , the correlation coefficient $\rho^{\prime \prime}\left(W_{n}, F_{n+1} \circ f_{n}\right)$ for current return $W_{n}(\cdot, v, x)$ and maximum final-period expected return $F_{n+1} \circ f_{n}(\cdot, v, x)$ attains its maximum value 1.0 when $H_{n+1}$ is positive linear affine. Thus, given that $H_{n+1}$ is increasing, a natural choice of inverse measure for positive correlation in returns is the maximum absolute 
magnitude

$$
\Psi \equiv \sup _{z \in \mathscr{A}}\left|H_{n+1}^{\prime \prime}(z)\right|
$$

of the second derivative $H_{n+1}^{\prime \prime}$ of $H_{n+1}$ over the range set

$$
\mathscr{R} \equiv\left\{z \in R \mid z=W_{n}(\omega, v, x) \text { for some }(\omega, v) \in \Omega \times V(n, x)\right\},
$$

for the current return function section $W_{n}(\cdot, \cdot, x)$.

The first theorem, below, demonstrates the appropriateness of this interpretation for $\Psi$, as well as pointing out a similar possible interpretation for the infimum

$$
\Pi \equiv \inf _{z \in \mathscr{T}} H_{n+1}^{\prime}(z)
$$

of the first derivative $H_{n+1}^{\prime}$ of $H_{n+1}$ over $\Re$. Specifically, the correlation coefficient $\rho^{\prime \prime}\left(W_{n}, F_{n+1} \circ f_{n}\right)$ is shown to vary inversely with $\Psi$ and directly with $\Pi$.

Theorem 4.1. Let $\Psi$ and $I 7$ be defined by (32) and (34). Then, for each admissible control $v \in V(n, x)$, the correlation coefficient $\rho^{\prime \prime}\left(W_{n}, F_{n+1} \circ f_{n}\right)$ is a positive number satisfying

$$
\left[\rho^{\prime \prime}\left(W_{n}, F_{n+1} \circ f_{n}\right)\right]^{2} \geqq 1-\frac{\Psi^{2} \sigma^{4}(v)}{4 I \eta^{2} \sigma^{2}(v)} .
$$

The next theorem provides an upper bound for the return loss (28) which varies directly with both the variance measure $\sigma^{2}$ for uncertainty and the inverse measure $\Psi$ for positive correlation in returns.

Theorem 4.2. The return loss (28) satisfies

$$
0 \leqq G\left(v^{\circ}\right)-G\left(v^{*}\right) \leqq a_{n}(x) \sigma^{2} \Psi .
$$

If $\mathrm{H}_{n+1}$ is either concale or convex, these bounds can be improved to

$$
0 \leqq G\left(v^{\circ}\right)-G\left(v^{*}\right) \leqq \frac{1}{2} a_{n}(x) \sigma^{2} \Psi .
$$

Theorem 4.2 characterizes the trade-off among uncertainty, positive correlation in returns, and global return loss. From a practical standpoint, the presence of $\Psi$ in the return loss bound is unfortunate; for the computation of its magnitude requires the recursive consideration of future 
return possibilities. However, any exact bound provided for the return loss (28) must necessarily involve such considerations.

The following question can nevertheless be posed: Even if $\Psi$ is not explicitly computed, might it be possible to guarantee an improvement of the bound in (36) by a more judicious selection of the intermediate return function $W_{n}$ ? For the problem at hand the objective of the planner is to maximize final-period expected return $E\left[W_{N}\left(\omega_{N}, v_{N}, x_{N}\right) \mid v, \bar{x}\right]$ via selection of an admissible feedback control law $\boldsymbol{v}=\left(v_{0}, \ldots, v_{N}\right)$. As discussed in section 3 , dynamic programming regularity conditions guarantee this objective can be achieved by the sequential maximization of $E_{n}\left[F_{n+1} \circ f_{n}\left(\omega_{n}, v_{n}, x_{n}\right) \mid v_{n}, x_{n}\right]$ with respect to $v_{n} \in V\left(n, x_{n}\right), n=0, \ldots, N$, where each return-to-go function $F_{n+1}$ depends only on $W_{N}$, the state functions $f_{n+1}, \ldots, f_{N}$, and the transitional probability distributions governing $\omega_{n+1}, \ldots, \omega_{N}$. The intermediate return functions $W_{1}, \ldots, W_{N-1}$ are thus only proxies for the optimal intermediate return functions $F_{2} \circ f_{1}, \ldots, F_{N} \circ f_{N-1}$, which are generally impossible to compute in closed form. What criteria might be used to select the 'best' proxy from among any given set of candidate intermediate return functions $W_{n}$ ?

The following theorem clarifies certain characteristics of intermediate return functions $W_{n}$ which partially order them in terms of global return performance. Let $Y$ denote the set

$$
Y \equiv\left\{y \in R \mid y=f_{n}(\omega, v, x) \text { for some }(\omega, v) \in \Omega \times V(n, x)\right\}
$$

and, for any twice differentiable function $Q: Y \rightarrow R$ with $Q^{\prime}>0$, let $R_{Q}: Y \rightarrow R$ denote the Pratt-Arrow measure of absolute risk aversion defined by $R_{Q}(y)$ $\equiv Q^{\prime \prime}(y) / Q^{\prime}(y)$. Interpreting $Q(\cdot)$ as a utility of wealth function for an expected utility maximizing decision maker, it can be shown [Pratt (1964, p. 125)] that $R_{Q}(y)$ is approximately equal to $2 \cdot\left(y, z^{*}\right) / \sigma_{z}^{2}$. for any zero-mean random variable $z^{*}$ with small variance $\sigma_{:}^{2}$, where the risk premium $r\left(y, z^{*}\right) \in R$ is the maximum amount the decision maker would be willing to pay to avoid a gamble on $z^{*}$; i.e., $r\left(y, z^{*}\right)$ satisfies $Q\left(y-r\left(y, z^{*}\right)\right)=E_{z} \cdot Q(y$ $+z)$. Thus, in principle, $R_{Q}(y)$ can be directly elicited by suitable gamble experiments even if $Q(\cdot)$ is unknown to the experimenter.

Theorem 4.3. Suppose the state space $X$ is a subset of $R$, the nth period return-to-go function $F_{n+1}: X \rightarrow R$ is strictly increasing, and the nth period intermediate return function $W_{n}: \Omega \times V \times X \rightarrow R$ is given by

$$
W_{n}(\omega, v, x)=U \circ f_{n}(\omega, v, x)=U\left(x_{n+1}\right) \text {, }
$$

for some strictly increasing twice differentiable function $U: X \rightarrow R$. If $S: X \rightarrow R$ 
is any other strictly increasing twice differentiable function satisfying

$$
\begin{aligned}
& \sigma^{2} \equiv \sup _{v \in V(n, x)} \operatorname{var}\left(U \circ f_{n}(\omega, v, x)\right) \\
& \quad>\sup _{v \in V(n, x)} \operatorname{var}\left(S \circ f_{n}(\omega, v, x)\right) \equiv \sigma_{S}^{2}, \\
& \frac{\left|R_{U}(y)-R_{F_{n+1}}(y)\right|}{\left[U^{\prime}(y)\right]^{2}} \geqq \frac{\left|R_{S}(y)-R_{F_{n+1}}(y)\right|}{\left[S^{\prime}(y)\right]^{2}}, \quad y \in Y,
\end{aligned}
$$

with $R_{U} \equiv R_{F_{N+1}}$, then the upper bound for the global return loss $G\left(v^{\circ}\right)-G\left(v^{*}\right)$ given in (36) can be strictly reduced if $v^{*}$ is replaced with any solution $v^{s}$ to

$$
\max _{v \in V(n, x)} E_{n}\left[S \circ f_{n}(\omega, v, x) \mid v, x\right] .
$$

The import of Theorem 4.3 is that absolute risk aversion, marginal return, and variance are the three crucial criteria for selecting among candidate intermediate return functions of the form (39). Sufficient conditions guaranteeing the monotonicity of $F_{n+1}$ are provided in Theorem 3.6.

\section{Illustrative examples}

The general macro policy and portfolio models outlined below, as examples of the basic model, were selected for their familiarity and common usage in economic analysis. Several researchers have discovered special cases of these models for which the optimal feedback control law reduces to a sequence of myopically optimal control selections. As the following discussion demonstrates, a systematic explanation for the occurrence of both exact and approximate myopic-global return equivalence within each of these models is provided by the results of sections 3 and 4 . Specifically, certain parameter configurations and function specifications yield linear positive correlation in period-by-period returns. Moreover, under weak restrictions one still obtains positive correlation in the sense of Theorem 3.6; hence, by Theorem 4.2, the global return loss associated with myopic optimization is bounded above by terms which vary directly with the degree of uncertainty and inversely with the degree of positive correlation in period-by-period returns.

Example 5.1. Dynamic linearized macro policy model with random coefficients [cf. Aoki (1976, 1967), Chow (1975)]. Dynamic macro policy models are commonly specified in linearized reduced form with random 
coefficients, as follows:

$$
\begin{aligned}
& x_{0}=\bar{x} \quad \text { (initial conditions), } \\
& x_{n+1}=A_{n} x_{n}+B_{n} z_{n}+C_{n} v_{n}+\varepsilon_{n}, \quad 0 \leqq n \leqq N,
\end{aligned}
$$

where $x_{n} \in R^{q}$ is a column vector of dependent state variables, $z_{n} \in R^{m}$ is a column vector of uncontrollable exogenous variables, $v_{n} \in R^{r}$ is a column vector of controllable exogenous variables, $\varepsilon_{n} \in R^{q}$ is a column vector of random disturbances, and $A_{n}, B_{n}$, and $C_{n}$ are random coefficient matrices with dimension $q \times q, q \times m$, and $q \times r$, respectively. If system (42) is a firstorder Taylor's approximation for some underlying nonlinear state equation, then the probability distribution governing the vector $\omega_{n}=\left(A_{n}, B_{n}, C_{n}, \varepsilon_{n}\right)$ of Jacobian and remainder terms may depend on the current state $x_{n}$ and control selection $v_{n}$ as well as on the current time $n$. Letting $A_{n} x_{n}+B_{n} z_{n}$ $+C_{n} v_{n}+\varepsilon_{n} \equiv f_{n}\left(\omega_{n}, v_{n}, x_{n}\right)$, and assuming the usual total expected return objective, with $n$th period return given by some continuous function $S_{n}\left(v_{n}, x_{n}\right.$, $\left.x_{n+1}\right)=U_{n}\left(v_{n}, x_{n}, f_{n}\left(\omega_{n}, v_{n}, x_{n}\right)\right) \equiv W_{n}\left(\omega_{n}, v_{n}, x_{n}\right)$ of the control and initial and final state for period $n$, this linearized macro model has the basic model format.

Consider, first, the special case in which the $n$th period intermediate return function $W_{n}(\cdot)$ is given by $W_{n}(\omega, v, x)=\left[U_{n} \circ f_{n}\right](\omega, v, x)=U_{n}\left(x_{n+1}\right)$ for some continuous non-decreasing utility function $U_{n}$ with well-defined inverse $g_{n}$ $\equiv U_{n}^{-1}$. In addition, suppose for each $n$ that the components of the coefficient matrix $A_{n}$ are non-negative with probability 1.0 , and the distribution for $\omega_{n}$ is independent of $x_{n}$. The hypotheses of Theorem 3.6 are then easily verified. In particular, one obtains

$$
T_{n+1} \circ f_{n}=T_{n+1} \circ U_{n}^{-1} \circ U_{n} \circ f_{n}=H_{n+1}^{* *} \circ W_{n},
$$

where $H_{n+1}^{* *} \equiv T_{n+1} \circ U_{n}^{-1}$ is non-decreasing. It follows by a simple modification of Theorem 4.2 that the global return loss resulting from myopic optimization within this macro policy model varies directly with the degree of uncertainty and inversely with the degree of positive correlation in period-by-period returns.

Consider once again the originally outlined basic format macro model. Suppose the components of $A_{n}, B_{n}, C_{n}$, and $\varepsilon_{n}$ are serially independent random variables distributed independently of $x_{n}$ and $v_{n}$, with finite first and second moments; the components of the matrices $A_{n}, B_{n}$, and $C_{n}$ are uncorrelated with the components of $\varepsilon_{n}$; and the components of $\varepsilon_{n}$ have zero mean. In addition, suppose $n$th period return is given by the quadratic 
specification,

$$
\begin{aligned}
W_{n}\left(\omega_{n}, v_{n}, x_{n}\right) & =k_{n}-f_{n}\left(\omega_{n}, v_{n}, x_{n}\right)^{T} K f_{n}\left(\omega_{n}, v_{n}, x_{n}\right) \\
& =k_{n}-x_{n+1}^{T} K x_{n+1},
\end{aligned}
$$

for some constant positive definite $q \times q$ matrix $K$ and some constant $k_{n} \in R$. Finally, letting $E_{n}[\cdot]$ denote expectation with respect to the distribution of $\omega_{n}$, suppose $\left|E_{n} C_{n}{ }^{T} K C_{n}\right| \neq 0, n \in\{0, \ldots, N\}$.

In order to have maximum expected return in period $n+1$ be a nondecreasing linear affine function of the return (43) realized in period $n$, in the sense of Corollary 3.3, it can be shown by straightforward calculation that it suffices to have

$$
E_{n+1}\left[A_{n+1}+C_{n+1} G_{n+1}\right]^{T} K\left[B_{n+1}+C_{n+1} g_{n+1}\right]=0,
$$

and

$$
E_{n+1}\left[A_{n+1}+C_{n+1} G_{n+1}\right]^{T} K\left[A_{n+1}+C_{n+1} G_{n+1}\right]=b_{n} K,
$$

for some $b_{n} \geqq 0$, where

$$
\begin{aligned}
& g_{n+1} \equiv-\left[E_{n+1} C_{n+1}^{T} K C_{n+1}\right]^{-1} E_{n+1} C_{n+1}^{T} K B_{n+1}, \\
& G_{n+1} \equiv-\left[E_{n+1} C_{n+1}^{T} K C_{n+1}\right]^{-1} E_{n+1} C_{n+1}^{T} K A_{n+1} .
\end{aligned}
$$

Given (44) and (45), it follows by Corollary 3.3 that the control selections

$$
v_{n}^{*}(x) \equiv G_{n+1} x_{n+1}+g_{n+1} z_{n+1},
$$

which sequentially maximize current expected return, also yield the optimal control selections for the global maximization of total expected return.

Condition (44) holds if and only if

$$
\begin{aligned}
E_{n+1} A_{n+1}^{T} K B_{n+1}= & \left(E_{n+1} A_{n+1}^{T} K C_{n+1}\right)\left(E_{n+1} C_{n+1}^{T} K C_{n+1}\right)^{-1} \\
& \times\left(E_{n+1} C_{n+1}^{T} K B_{n+1}\right) .
\end{aligned}
$$

Various restrictions imply (49): e.g., $C_{n+1}$ a constant non-singular matrix, and $A_{n+1}$ uncorrelated with $B_{n+1} ; B_{n+1}=C_{n+1} ; A_{n+1}=C_{n+1} ; A_{n+1}=0$; or $B_{n+1}=0$. Condition (45) holds with $b_{n}=0$ if and only if

$$
\begin{aligned}
E_{n+1} A_{n+1}^{T} K A_{n+1}= & \left(E_{n+1} A_{n+1}^{T} K C_{n+1}\right)\left(E_{n+1} C_{n+1}^{T} K C_{n+1}\right)^{-1} \\
& \times\left(E_{n+1} C_{n+1}^{T} K A_{n+1}\right) .
\end{aligned}
$$


Again, various restrictions imply (50): e.g., $A_{n+1}$ and $C_{n+1}$ constant nonsingular matrices; $A_{n+1}=C_{n+1}$; or $A_{n+1}=0$. Finally, condition (45) always holds if the dimension $q$ of the matrix $K$ is 1 .

Aoki (1967) notes the equivalence of global and myopic optimization only for the special case $B_{n+1}=0$ and $q=1$. Chow (1975) does not discuss the issue.

Example 5.2. Portfolio model [cf. Arrow (1971), Mossin (1968), Hakansson (1971), Bellman and Kalaba (1957), and Kalaba and Tesfatsion (1978)]. In each period $n \in\{0, \ldots, N\}$ an investor must decide how to allocate his current wealth $x_{n} \in X \subseteq R_{+}$between two investment opportunities $A$ and $B$, the first yielding a positive or negative net return rate $\pm s_{n} \quad\left(0<s_{n} \leqq 1\right)$ with probabilities $p_{n}$ and $1-p_{n}$, and the second yielding a net return rate $r_{n}\left(0 \leqq r_{n}\right.$ $<s_{n}$ ) with probability 1 . The investor's objective is to maximize the expected utility of his wealth $x_{N+1}$ at the end of period $N$ via feedback control.

Assuming the investor's initial wealth $x_{0}$ for period 0 is positive, his initial wealth $x_{n+1}$ for period $n+1$ is a simple function of his initial wealth $x_{n}$ for period $n$, the net return rate $\omega_{n} \in\left\{s_{n},-s_{n}\right\}$ observed for investment opportunity $A$ in period $n$, and the amount $v_{n} \in\left[0, x_{n}\right] \equiv V\left(n, x_{n}\right)$ of wealth he allocated to $A$ in period $n$; namely $x_{n+1}=x_{n}+\omega_{n} v_{n}+r_{n}\left[x_{n}-v_{n}\right] \equiv f_{n}\left(\omega_{n}, v_{n}\right.$, $x_{n}$ ). Assuming utility of wealth at the end of period $n, n \in\{0, \ldots, N\}$, is measured by $W_{n}\left(\omega_{n}, v_{n}, x_{n}\right) \equiv U_{n}\left(x_{n}+\omega_{n} v_{n}+r_{n}\left[x_{n}-v_{n}\right]\right)=U_{n}\left(x_{n+1}\right)$, where $U_{n}: X \rightarrow R$ is a continuous strictly increasing function of $x$, this portfolio problem has the basic model format with final-period expected wealth objective.

Let $U_{n}^{-1}$ denote the strictly increasing inverse function for $U_{n}$. It is then easily verified that all of the hypotheses of Theorem 3.3 hold with $g_{n} \equiv U_{n}^{-1}$. In particular, since $W_{n}(\omega, v, x)$ is strictly increasing in $x$, one obtains $F_{n+1} \circ f_{n}$ $=F_{n+1} \circ U_{n}^{-1} \circ U_{n} \circ f_{n}=H_{n+1}^{*} \circ W_{n}$, where $H_{n+1}^{*} \equiv F_{n+1} \circ U_{n}^{-1}$ is strictly increasing. It follows by Theorem 4.2 that the global return loss resulting from myopic optimization within this portfolio model is bounded above by terms which vary directly with the degree of uncertainty and inversely with the degree of positive correlation in period-by-period returns.

Consider now the special case in which $U_{n}(x) \equiv \log (x), x \in X \equiv R_{++}$. Letting $E_{n}[\cdot]$ denote expectation with respect to the distribution of the net return rate $\omega_{n} \in\left\{-s_{n}, s_{n}\right\}$, the control $v^{*} \equiv v_{n+1}^{*}(x) \in[0, x]$ which maximizes expected utility of wealth $E_{n+1} \log \left(x_{n+2}\right)$ for period $n+1$, beginning in the initial wealth state $x$, is then given by

$$
\begin{aligned}
& v^{*}=0 \quad \text { if } \quad D<0 \text {, } \\
& =D x \text { if } 0 \leqq D \leqq 1 \text {, } \\
& =x \text { if } 1<D \text {, }
\end{aligned}
$$


where

$$
D \equiv \frac{[1+r][2 p s-s-r]}{[s+r][s-r]}
$$

and time subscripts have been dropped on $r_{n+1}, s_{n+1}$, and $p_{n+1}$ to ease notation. Maximum expected utility of wealth for period $n+1$ beginning in state $x$ is then given by

$$
\begin{aligned}
M_{n+1}(x)= & \log (x)+p \log (1+r+[s-r] d) \\
& +[1-p] \log (1+r-[s+r] d),
\end{aligned}
$$

where $d \equiv v^{*}(x) / x$. Thus, $M_{n+1}(x)$ is a positive linear affine function of the return $\log (x)$ realized in period $n$ in the sense of Corollary 3.3. It follows that the myopic control selections (51) yield an optimal feedback control law for the investor's final-period wealth objective. Using a different line of reasoning requiring the existence of an interior regular optimal control law, Mossin (1968) and Hakansson (1971) reach a similar conclusion; and Kalaba and Tesfatsion (1978) reach a similar conclusion for the special case $r_{n}=0$, $n \in\{0, \ldots, N\}$. The issue is not discussed in Arrow (1971) and Bellman and Kalaba (1957).

\section{Discussion}

Many years ago $H$. Simon introduced the concept of 'satisficing' to describe the process by which boundedly rational decision makers ultimately select, implement, and evaluate their actions. An apt illustration of satisficing would seem to be the chess player, myopically attempting to achieve his global checkmate objective through the sequential realization of intermediate player-piece configurations. Is it possible, in any nontrivial context, to analytically investigate the decomposition of global objectives into sequences of myopic intermediate objectives, so that some conclusions may be drawn regarding the 'best' selection of these intermediate objectives?

A cautiously affirmative answer is provided in the present paper. Within the context of a stochastic control model encompassing many well-known micro and macro models, three specific questions are posed:

Under what conditions will the myopic sequential maximization of expected current return result in optimal global expected return performance?

More generally, what is the global expected return loss associated with the use of myopic sequential expected return maximization, and how does it vary with increases in uncertainty and positive correlation in period-by-period returns? 
What characteristics of intermediate-period return functions allow them to be partially ordered in terms of final-period expected return performance?

The basic answer presented for the first question, Theorems 3.1 and 3.2, provides a systematic explanation for the occurrence of myopic and global return equivalence in a variety of economic models. The basic answer provided for the second question, Theorem 4.2, characterizes the trade-off among uncertainty, positive correlation in returns, and global return loss. In answer to question three, Theorem 4.3 demonstrates that the correlation structure of the intermediate objectives, the basic environmental uncertainty reflected by intermediate return variance, and the relative degree of absolute risk aversion reflected by intermediate and primitive (optimality equation) return functions are three crucial criteria for ordering alternative intermediate-period return function specifications in terms of final-period expected return performance.

A further aspect of the results presented in Sections 3 and 4 which has not been explored in the present paper is their possible use for estimating the global expected return loss resulting from the return-to-go approximations suggested by previous researchers, e.g., Aoki (1976), Bar-Shalom and Tse (1976), Chow (1975), and Norman (1976). As explained in footnote 8, returnto-go approximation problems can be viewed as myopic-global problems under a simple transformation of the original period-by-period return functions.

\section{Appendix: Proof outlines}

A.1. Proof of Theorem 3.1. Given condition (15), it can be verified that each of the restriction sets (1) through (5) guarantees $E_{n}\left\lfloor W_{n} \mid v, x_{n}\right\rfloor$ and $E_{n}\left[F_{n+1} \circ f_{n} \mid v, x_{n}\right]=a_{n}\left(x_{n}\right) E_{n}\left[H_{n+1} \circ W_{n} \mid v, x_{n}\right]+b_{n}\left(x_{n}\right)$ have the same set of maximizing controls $v \in V\left(n, x_{n}\right)$ for each $n \in\{0, \ldots, N-1\}$. Thus a feedback control law $v^{*} \in \mathscr{L}$ satisfies the myopic expected return objective (4) if and only if the equalities (9) hold, i.e., if and only if $\boldsymbol{v}^{*}$ is also an optimal feedback control law for the final-period expected return objective (5). The final assertion of Theorem 3.1 is easily verified using analogous arguments. Q.E.D.

A.2. Proof of Corollary 3.3. By induction, condition (18) implies that $\left[F_{n+1} \circ f_{n}\right](\omega, v, x)=y_{n}^{\prime}(x) W_{n}(\omega, \quad v, x)+z_{n}^{\prime}(x)$ and $\left[T_{n+1} \circ f_{n}\right](\omega, \quad v, x)$ $=y_{n}^{\prime \prime}(x) W_{n}(w, v, x)+z_{n}^{\prime \prime}(x)$ for some functions $y_{n}^{\prime}: X \rightarrow R_{++}, z_{n}^{\prime \prime}: X \rightarrow R, y_{n}^{\prime \prime}: X$ $\rightarrow R_{++}$, and $z_{n}^{\prime \prime}: X \rightarrow R$ for each $n \in\{0, \ldots, N-1\}$. Thus conditions (15) and (16) in Theorems 3.1 and 3.2 both hold for positive linear functions $H_{n+1}$. The first assertion of Corollary 3.3 follows. If the functions $c_{n}(\cdot)$ in (18) take 
on zero values, then the functions $y_{n}^{\prime}$ and $y_{n}^{\prime \prime}$ may also take on zero values. By Theorems 3.1 and 3.2, the equivalence between objectives (4) and (5) need no longer hold but the equivalence between objectives (4) and (6) is unaffected. If the functions $d_{n}(\cdot)$ in (18) depend on $\omega$, then the functions $y_{n}^{\prime}$ and $y_{n}^{\prime \prime}$ will also depend on $\omega$. As long as each distribution $p_{n}\left(\cdot \mid v_{n}, x_{n}\right)$, $n \in\{0, \ldots, N-1\}$, is independent of the selected control $v_{n}$, Theorems 3.1 and 3.2 still yield the desired conditions. Q.E.D.

A.3. Proof of Lemma 3.4. Let $n=N$. By definition, $F_{N}(x)=E_{N}\left[\varphi \circ f_{N}(\omega\right.$, $\left.\left.v_{N}^{\circ}(x), x\right)\right]$. By compactness of $\Omega$ and twice continuous differentiability of $\varphi(\cdot)$ and $f_{n}(\omega, \cdot)$, it follows by a Lebesgue dominated convergence argument that expectation and differentiation operations can be interchanged up to the second order. Thus the first- and second-order sufficient conditions for a maximum, satisfied by $v_{N}^{o}(x)$ by assumption (A.3), imply (20a) and (22b) hold with negative definite $D_{N}(x)$. It follows by an implicit function argument that $v_{N}^{\circ}(x)$ is continuously differentiable in a neighborhood of $x$, and satisfies (21). The derivatives (20b) and (20c) then follow by direct calculation. The analogous results for $n<N$ are similarly obtained. Q.E.D.

The following theorem can be proved using Theorem 3.1, restriction set (1), but a much simpler proof is obtained by using the later independent Theorem 4.2. The latter proof is indicated here.

A.4. Proof of Theorem 3.5. Let $n=N$ and $R_{\varphi}(x) \equiv c$ for some $c \in R$. By Lemma 3.4,

$$
\begin{aligned}
F_{N}^{\prime}(x) & =b_{N} E_{N}\left[\varphi^{\prime}\left(f_{N}(d)\right)\right] . \\
F_{N}^{\prime \prime}(x) & =b_{N} E_{N}\left[\varphi^{\prime \prime}\left(f_{N}(d)\right) \frac{\partial f_{N}}{\partial v}(d)\right] \frac{\partial v_{N}^{o}}{\partial x}(x)+b_{N}^{2} E_{N}\left[\varphi^{\prime \prime}\left(f_{N}(d)\right)\right] \\
& =-b_{N} c E_{N}\left[\varphi^{\prime}\left(f_{N}(d)\right) \frac{\partial f_{N}^{\prime}}{\partial v}(d)\right] \frac{\partial v_{N}^{o}}{\partial x}(x)-b_{N}^{2} c E_{N}\left[\varphi^{\prime}\left(f_{N}(d)\right)\right] \\
& =0-b_{N}^{2} c E_{N}\left[\varphi^{\prime}\left(f_{N}(d)\right)\right] .
\end{aligned}
$$

Thus $R_{F_{N}}(y) \equiv-F_{N}^{\prime \prime}(y) / F_{N}^{\prime}(y)=b_{N} c$ for each $y \in Y$, where $Y$ is defined in (38). It follows by induction that $R_{F_{n+1}}(y)=\left(\Pi_{j=1}^{N-n} b_{n+j}\right) c, y \in Y$. The function $U: X$ $\rightarrow R$ defined in Theorem 3.5 satisfies $R_{U}(y)=\left(\Pi_{j=1}^{N-n} b_{n+j}\right) c, y \in Y$, hence the desired conclusion follows from Lemma 3.4 and Theorem 4.2 with $W_{n}(\omega, v, x)$ $\equiv U \circ f_{n}(\omega, v, x)$ by noting that each $z \equiv W_{n}(\omega, v, x)$ has the form $U(y)$ for some $y \in Y$, and

$$
H_{n+1}^{\prime \prime}(U(y))=\frac{F_{n+1}^{\prime}(y)}{\left[U^{\prime}(y)\right]^{2}}\left[R_{U}(y)-R_{F_{n+1}}(y)\right]
$$


is zero for each $y \in Y$, where $H_{n+1} \equiv F_{n+1} \circ U^{-1}$ satisfies $H_{n+1} \circ W_{n}=F_{n+1} \circ f_{n}$ and $H_{n+1}^{\prime}(x)=F_{n+1}^{\prime}\left(U^{-1}(x)\right) U^{-1 \prime}(x)>0, x \in R$. Q.E.D.

A.5. Proof of Theorem 3.6. The proof will be indicated for the return-to-go functions $T_{n+1}$ in the non-decreasing case. Proof of the remaining assertions can then be obtained by minor modifications.

By a well-known result in the stochastic dominance literature [see, e.g., Tesfatsion (1976)], if $\Omega \subset R$, then, by the stochastic dominance restriction (3),

$$
x^{\prime \prime} \geqq x^{\prime} \Rightarrow E_{N}\left[U\left(\omega, x^{\prime \prime}\right) \mid v, x^{\prime \prime}\right] \geqq E_{N}\left[U\left(\omega, x^{\prime \prime}\right) \mid v, x^{\prime}\right],
$$

for all continuous functions $U: \Omega \times X \rightarrow R$ which are non-decreasing with respect to $\omega \in \Omega$. Clearly (A.2) holds trivially if $p_{N}(\cdot \mid v, x)$ is independent of $x$. If (A.2) holds and $U(\omega, x)$ is non-decreasing with respect to $x$, then $x^{\prime \prime} \geqq x^{\prime}$ $\Rightarrow E_{N}\left[U\left(\omega, x^{\prime \prime}\right) \mid v, x^{\prime \prime}\right] \geqq E_{N}\left[U\left(\omega, x^{\prime}\right) \mid v, x^{\prime}\right]$. Thus restrictions (2), (3), and (4) imply that $E_{N}\left[W_{N}(\omega, v, x) \mid v, x\right]$ is non-decreasing in $x$ for each $v \in V$. It follows by restriction (5) that $T_{N}(x)$ is non-decreasing in $x$.

Now suppose $T_{n+1}: X \rightarrow R$ is non-decreasing for some $n \in\{0, \ldots, N-1\}$. Restrictions (1) and (4) then imply $\left[T_{n+1} \circ f_{n}\right](\omega, v, x)=\left[T_{n+1} \circ g_{n} \circ W_{n}\right](\omega, v, x)$ is non-decreasing in $\omega$ and $x$ for all $v \in V$. It follows by restrictions (2) and (3) that $E_{n}\left[W_{n}(\omega, v, x)+T_{n+1} \circ f_{n}(\omega, v, x) \mid v, x\right]$ is non-decreasing in $x$ for all $v \in V$, hence, by restriction (5), the same holds true for $T_{n}(x)$.

By induction, $T_{n+1}: X \rightarrow R$ is non-decreasing for all $n \in\{0, \ldots, N-1\}$; and, by restriction (1), the same is true for $H_{n+1}^{* *} \equiv T_{n+1} \circ g_{n}$. Q.E.D.

A.6. Proof of Theorem 4.1. Condition (15), assumed to hold for $H_{n+1}$, implies that $\rho^{v}\left(W_{n}, F_{n+1} \circ f_{n}\right)=\rho^{v}\left(W_{n}, H_{n+1} \circ W_{n}\right)$; and $\rho^{v}\left(W_{n}, H_{n+1} \circ W_{n}\right) \in(0,1]$ by Theorem 1 in Hildreth-Tesfatsion (1977).

By Taylor's Theorem, for each $\omega \in \Omega$ there exists $\Delta \equiv \Delta(\omega, v, x)$ and $\theta \equiv$ $\theta(\omega, v, x)$ lying between $W_{n}(\omega, v, x)$ and expected current return $M(v)$ such that

$$
\begin{aligned}
H_{n+1} \circ W_{n}(\omega, v, x)= & H_{\cdot n+1}(M(v))+H_{n+1}^{\prime}(M(v))\left[W_{n}(\omega, v, x)-M(v)\right] \\
& +\frac{1}{2} H_{n+1}^{\prime \prime}(\Delta)\left[W_{n}(\omega, v, x)-M(v)\right]^{2},
\end{aligned}
$$

and

$$
H_{n+1} \circ W_{n}(\omega, v, x)=H_{n+1}(M(v))+H_{n+1}^{\prime}(\theta)\left[W_{n}(\omega, v, x)-M(v)\right]
$$

Using (A.3),

$$
\begin{aligned}
{\left[\rho^{v}\left(W_{n}, H_{n+1} \circ W_{n}\right)\right]^{2} } & =[B+C]^{2} / D \\
& =\left\{D-\left[\sigma^{2}(v) \operatorname{var}(A)-C^{2}\right]\right\} / D,
\end{aligned}
$$


where

$$
\begin{aligned}
& A \equiv \frac{1}{2} H_{n+1}^{\prime \prime}(\Delta)\left[W_{n}(\omega, v, x)=M(v)\right]^{2}, \\
& B \equiv H_{n+1}^{\prime}(M(v)) \sigma^{2}(v), \\
& C \equiv E_{n}\left[\left(W_{n}(\omega, v, x)-M(v)\right) A \mid v, x\right] \\
& D \equiv B^{2}+2 B C+\sigma^{2}(v) \operatorname{var}(A)=\operatorname{var}\left(W_{n}\right) \operatorname{var}\left(H_{n+1} \circ W_{n}\right),
\end{aligned}
$$

and

$$
\operatorname{var}(A) \equiv E_{n}\left[\left(A-E_{n}[A \mid v, x]\right)^{2} \mid v, x\right]
$$

Using Hölder's Inequality and definitions (A.6)-(A.10),

$$
0 \leqq C^{2} \leqq \sigma^{2}(v) \operatorname{var}(A) \leqq \frac{1}{4} \sigma^{2}(v) \sigma^{4}(v) \Psi^{2},
$$

and

$$
D \geqq[B+C]^{2}=\left[\operatorname{cov}\left(W_{n}, H_{n+1} \circ W_{n}\right)\right]^{2} .
$$

Using the first-order Taylor expansion (A.4),

$$
\operatorname{cov}\left(W_{n}, H_{n+1} \circ W_{n}\right) \geqq \sigma^{2}(v) \Pi .
$$

Combining (A.11) through (A.13) with (A.5), one obtains the desired relation (35). Q.E.D.

A.7. Proof of Theorem 4.2. Using definition (25) for $G(v)$ and the Taylor expansion (A.3),

$$
\begin{aligned}
G(v)= & a_{n}(x) H_{n+1}(M(v))+b_{n}(x) \\
& +\frac{1}{2} a_{n}(x) E_{n}\left[H_{n+1}^{\prime \prime}(\Delta)\left(W_{n}(\omega, v, x)-M(v)\right)^{2} \mid v, x\right] .
\end{aligned}
$$

By definition, $v^{*}$ maximizes expected current return $M(v)$ over $V(n, x)$. Since $H_{n+1}$ is increasing, $H_{n+1}\left(M\left(v^{\circ}\right)\right) \leqq H_{n+1}\left(M\left(v^{*}\right)\right)$. Also by definition, $v^{\circ}$ maximizes $G(v)$ over $V(n, x)$. Thus

$$
\begin{aligned}
0 \leqq & G\left(v^{\mathrm{o}}\right)-G\left(v^{*}\right) \\
\leqq & \frac{1}{2} a_{n}(x) E_{n}\left[H_{n+1}^{\prime \prime}\left(\Delta^{\mathrm{o}}\right)\left(W_{n}\left(\omega, v^{\mathrm{o}}, x\right)-M\left(v^{\mathrm{o}}\right)\right)^{2} \mid v^{\mathrm{o}}, x\right] \\
& -\frac{1}{2} a_{n}(x) E_{n}\left[H_{n+1}^{\prime \prime}\left(\Delta^{*}\right)\left(W_{n}\left(\omega, v^{*}, x\right)-M\left(v^{*}\right)\right)^{2} \mid v^{*}, x\right] \\
\leqq & \frac{1}{2} a_{n}(x)\left[\sigma^{2}\left(v^{\mathrm{o}}\right)+\sigma^{2}\left(v^{*}\right)\right] \Psi .
\end{aligned}
$$

The first claim of Theorem 4.2 then follows from (A.14) using definition (31) 
for $\sigma^{2}$; and the second claim follows from (A.14) by noting that concavity (convexity) of $H_{n+1}$ implies $H_{n+1}^{\prime \prime} \leqq(\geqq) 0$, thus the terms involving $v^{\circ}\left(v^{*}\right)$ in (A.14) can be discarded. Q.E.D.

A.8. Proof of Theorem 4.3. Under the assumptions of Theorem 4.3, the positive correlation condition (15) holds for each of the intermediate return functions $W_{n} \equiv U \circ f_{n}$ and $W_{n}^{S} \equiv S \circ f_{n}$, with $H_{n+1}(z) \equiv F_{n+1} \circ U^{-1}(z)$ and $H_{n+1}(z) \equiv F_{n+1} \circ S^{-1}(z), z \in \mathscr{R}$. Moreover, each $z \in \mathscr{R}$ has the representation $U(y)$ for some $y \in Y$.

By Theorem 4.2, $G\left(v^{\circ}\right)-G\left(v^{*}\right) \leqq \sigma^{2} \Psi$ and $G\left(v^{\circ}\right)-G\left(v^{s}\right) \leqq \sigma_{s}^{2} \Psi_{s}$, where $\Psi_{s}$ $\equiv \sup \left\{H_{n+1}^{S^{\prime \prime}}(z) \mid z=S(y)\right.$ for some $\left.y \in Y\right\}$. The difference $\sigma^{2} \Psi-\sigma_{S}^{2} \Psi_{S}$ is given by $\left[\sigma^{2}-\sigma_{S}^{2}\right] \Psi+\left[\Psi-\Psi_{S}\right] \sigma_{S}^{2}$. By (40a) and $(40 \mathrm{~b}), \sigma^{2}>\sigma_{S}^{2}$ and $\Psi>0$. To demonstrate that use of $S$ in place of $U$ reduces the global return loss bound in (36), it therefore suffices to establish that $\Psi \geqq \Psi_{S}$, which in turn holds if $\left|H_{n+1}^{\prime \prime}(U(y))\right| \geqq\left|H_{n+1}^{S \prime \prime}(U(y))\right|$ for all $y \in Y$. Using (A.1), the latter inequality follows directly from $(40 \mathrm{~b})$. Q.E.D.

\section{References}

Aoki, M., 1976, Optimal control and system theory in dynamic economic analysis (NorthHolland, Amsterdam).

Aoki, M., 1967, Optimization of stochastic systems (Academic Press, New York).

Arrow, K.J., 1971, Essays in the theory of risk bearing (Markham, Chicago, IL).

Bar-Shalom, Y. and E. Tse, 1976, Caution, probing, and the value of information in the control of uncertain systems, Annals of Economic and Social Measurement 5, 323-337.

Bellman, R., 1957, Dynamic programming (Princeton University Press, Princeton, NJ).

Bellman, R. and R.E. Kalaba, 1957, On the role of dynamic programming in statistical communication theory, IRE Transactions on Information Theory IT-3, 197-203.

Chow, G., 1975, Analysis and control of dynamic economic systems (Wiley, New York).

Day, R.H., 1969, Flexible utility and myopic expectations in economic growth, Oxford Economic Papers 21, 299-311.

Day, R.H. and Y.-K. Fan, 1976, Myopic optimizing, economic growth and the golden rule, Hong Kong Economic Papers 10, 12-20.

Hakansson, N., 1971, On optimal portfolio policies with and without serial correlation of yields, Journal of Business 44, 324-334.

Hildreth, C., 1974, Expected utility of uncertain ventures, Journal of the American Statistical Association 69, 9-17.

Hildreth, C. and L. Tesfatsion, 1977, A note on dependence between a venture and a current prospect, Journal of Economic Theory 15, 381-391.

Hinderer, K., 1971, Foundations of nonstationary dynamic programming with discrete time parameter (Springer-Verlag, New York).

Kalaba, R.E. and L. Tesfatsion, 1978, Two solution techniques for adaptive reinvestment: A small sample comparison, Journal of Cybernetics 8, $101-111$.

Keeler, E., 1974, Horizon in a simple model of economic growth, Econometrica 42, 273-277.

Koopmans, T.C., 1967, Objectives, constraints, and outcomes in optimal growth models, Econometrica 35, 1-15.

Landau, H.J., 1976, Pricing in a dynamic model with saturation, Econometrica 44, 1153-1155.

Leland, H.E., 1972, On the existence of optimal policies under uncertainty, Journal of Economic Theory 4, 35-44.

Leontief, W., 1958, Theoretical note on time-preference, productivity of capital, stagnation, and economic growth, American Economic Review XLVIII, 105-111. 
Leontief, W., 1959, Time preference and economic growth: Reply, American Economic Review XLIX, 1041-1043.

Levy, H. and J, Paroush, 1974, Toward multivariate efficiency criteria, Journal of Economic Theory $7,129-142$.

Kos, J., 1971, The approximate horizon in von Neumann models of optimal growth, in: Recent contributions to the von Neumann growth model (Springer-Verlag, Wien).

Mossin, J., 1968, Optimal multiperiod portfolio policies, Journal of Business 41, 215-229.

Norman, A.L., 1976, First order dual control, Annals of Economic and Social Measurement 5, 311-321.

Pitchford, J.D. and S.J. Turnovsky, 1977, Applications of control theory to economic analysis (North-Holland, Amsterdam).

Pratt, J., 1964, Risk aversion in the small and in the large. Econometrica 32, 122-136.

Simon, H., 1971, Models of bounded rationality, in: C.B. McGuire and R. Radner, eds., Decision and organization (North-Holland, Amsterdam).

Tesfatsion. L., 1976, Stochastic dominance and the maximization of expected utility, Review of Economic Studies XLIII, 301-315.

Tesfatsion, L., 1978, A new approach to filtering and adaptive control, Journal of Optimization Theory and Applications 25, 247-261.

Tesfatsion, L., 1979, Direct updating of intertemporal criterion functions for a class of adaptive control problems, IEEE Transactions on Systems, Man, and Cybernetics SMC-9, 143-151.

Tesfatsion, L., A conditional expected utility model for myopic decision makers. Theory and Decision, forthcoming.

Wilkes, S.S., 1962, Mathematical statistics (Wiley, New York). 